PROCEEDINGS OF THE

AMERICAN MATHEMATICAL SOCIETY

Volume 139, Number 4, April 2011, Pages 1221-1235

S 0002-9939(2010)10773-6

Article electronically published on November 4, 2010

\title{
THE EXISTENCE OF HYPERELLIPTIC FIBRATIONS WITH SLOPE FOUR AND HIGH RELATIVE EULER-POINCARÉ CHARACTERISTIC
}

\author{
HIROTAKA ISHIDA \\ (Communicated by Ted Chinburg)
}

\begin{abstract}
For any relatively minimal hyperelliptic fibration $f$ with slope four, there exists the inequality with respect to the relative Euler-Poincaré characteristic $\chi(f)$ of $f$ and the genus $g(f)$ of a fiber of $f$. This inequality restricts the extent of pairs $(g(f), \chi(f))$ for relatively minimal hyperelliptic fibrations $f$ with slope four which exist. Hence, for given suitable integers $g$ and $z$, we consider the existence of a relatively minimal hyperelliptic fibration $f$ with $g(f)=g, \chi(f)=z$ and slope four. The main purpose in this paper, for any positive integer $g$, is to prove that there exists a relatively minimal hyperelliptic fibration $f$ with $g(f)=g, \chi(f) \geq z(g)$ and slope four, where $z(X)$ is a certain polynomial of degree two.
\end{abstract}

\section{INTRODUCTION}

In this article, all varieties are defined over the complex number field. Let $f: X \longrightarrow C$ be a fibration from an algebraic surface $X$ of general type onto a smooth algebraic curve $C$ with genus $g(C)$. Denote the genus of a general fiber of $f$ by $g(f)$ and put $\Delta=(g(f)-1)(g(C)-1)$.

If $f$ is locally trivial, then we see the following numerical properties:

$$
\chi\left(\mathcal{O}_{x}\right)=\Delta, \quad K_{X}^{2}=8 \Delta, \quad e(X)=4 \Delta,
$$

where $K_{\bullet}, \chi\left(\mathcal{O}_{\bullet}\right)$ and $e(\bullet)$ denote a canonical divisor, the Euler-Poincaré characteristic and the topological Euler number of $\bullet$.

Let $K_{X / C}$ be the relative canonical divisor of $f$. We call $\chi(f)=\operatorname{deg} f_{*} K_{X / C}$ the relative Euler-Poincaré characteristic of $f$. Then discrepancies between invariants of $f$ and those of a locally trivial fibration can be computed by the following equations:

$$
\begin{aligned}
\chi(f) & =\chi\left(\mathcal{O}_{X}\right)-\Delta, \\
K_{X / C}^{2} & =K_{X}^{2}-8 \Delta, \\
e_{f} & =e(X)-4 \Delta,
\end{aligned}
$$

Received by the editors February 17, 2009 and, in revised form, October 14, 2009 and April 20, 2010

2010 Mathematics Subject Classification. Primary 14D06; Secondary 14J29.

This research was partly supported by the research grant 19740022 from JSPS.

(C)2010 American Mathematical Society 
where $e_{f}$ is computed as $\sum_{P \in C}\left\{e\left(f^{-1}(P)\right)+2 g(f)-2\right\}$. In fact, this sum is taken over all points $P$ such that $f^{-1}(P)$ are singular fibers. (If $f^{-1}(P)$ is nonsingular, then $e\left(f^{-1}(P)\right)=2-2 g(f)$.)

We assume that $f$ is relatively minimal and not locally trivial. In this case, we have $\chi(f) \neq 0([2$, III,Theorem 17.3]). We define the slope $\lambda(f)$ of $f$ to be $\lambda(f)=K_{X / C}^{2} / \chi(f)$. Note that $K_{X}^{2}=\lambda(f) \chi\left(\mathcal{O}_{X}\right)+(8-\lambda(f)) \Delta$. Then, we have the slope inequality $4(g(f)-1) / g(f) \leq \lambda(f)$, which is proved by Xiao [9, Theorem 2]. (Horikawa [4, V, Theorem 2.1] and Persson [8, Proposition 2.12] proved the slope inequality for hyperelliptic fibrations.) If we assume that $\lambda(f)<4$, then we have $g(f) \leq 4 /(4-\lambda(f))$; i.e., there exists the upper bound of $g(f)$ for any fibrations $f$ with $\lambda(f)<4$.

Furthermore, by Konno [6, Theorem 4.3], if almost all fibers of $f$ are nonhyperelliptic curves and $f_{*} K_{X / C}$ is semi-stable, then $\lambda(f) \geq(5 g(f)-6) / g(f)$. In particular, there exists the upper bound of $g(f)$ for any fibrations $f$ satisfying $\lambda(f)<5$ and the above assumptions. A fibration $f: X \longrightarrow C$ is called a hyperelliptic fibration if and only if almost all fibers of $f$ are hyperelliptic curves. From these slope inequalities, for any hyperelliptic fibrations $f$ with $4 \leq \lambda(f)<5$, the genus $g(f)$ may not be restricted. In order to show that there exists no restriction for $g(f)$, the author has studied hyperelliptic fibrations with slope four in [5] and proved the following:

Theorem 0.1 ([5, Theorem 0.1]). Let g be an integer greater than three. We set

$$
\Delta(g)= \begin{cases}\frac{g}{2} & \text { if } g \text { is even }, \\ g-3 & \text { if } g \text { is odd. }\end{cases}
$$

Then any relatively minimal hyperelliptic fibration $f: X \longrightarrow C$ with $g(f)=g$ and $\lambda(f)=4$ satisfies $\chi(f) \geq \Delta(g)$. Moreover, there exists a relatively minimal hyperelliptic fibration $f$ of genus $g$ with $\lambda(f)=4$ and $\chi(f)=\Delta(g)$.

From the above theorem, we see that there exists a restriction for $\chi(f)$. Our interest is the following problem: for any pair $(g, z)$ of suitable integers, does there exist a relatively minimal hyperelliptic fibration $f$ with $\lambda(f)=4, g(f)=g$ and $\chi(f)=z$ ? If we only consider fibrations $f$ with constant slope $\lambda$, then $X$ satisfies the numerical property $K_{X}^{2}=\lambda \chi\left(\mathcal{O}_{X}\right)+(8-\lambda) \Delta$. In particular, in our assumption, we have $K_{X}^{2}=4 \chi\left(\mathcal{O}_{X}\right)+4 \Delta$. Hence surfaces with such fibrations correspond to points $\left(\chi\left(\mathcal{O}_{X}\right), K_{X}^{2}\right)$ with $K_{X}^{2}=4 \chi\left(\mathcal{O}_{X}\right)+4 \Delta$ on the ordinary surface geography.

From [5, Corollary 2.9] and [8, Section 3], we obtained the following:

Corollary 0.2 (cf. [5, Corollary 2.9]). For any positive integer $z$, there exists a relatively minimal hyperelliptic fibration $f$ with $\lambda(f)=4, \chi(f)=z$ and $g(f)=$ $2,3,4$.

Corollary 0.2 gives an answer to the above problem in the case that $g(f)=$ $2,3,4$. Hence we consider the case that $g(f) \geq 5$. The main purpose of this paper is to prove the following:

Theorem 0.3. For any integers $g$ and $z$ satisfying one of the following conditions (i) and (ii), there exists a relatively minimal hyperelliptic fibration $f$ with $\lambda(f)=$ $4, g(f)=g$ and $\chi(f)=z$. 
(i) $g$ is an even integer which is greater than four and $z \geq g^{2}+\frac{g}{2}-2$,

(ii) $g$ is an odd integer which is greater than three and $z \geq g^{2}-1$.

By the above theorem, we see that there exists a relatively minimal hyperelliptic fibration $f$ with $g(f)=g, \chi(f) \gg 0$ and $\lambda(f)=4$ for any positive integer $g$. This gives an answer to our problem in the case of a fibration with high relative Euler-Poincaré characteristic.

If $f$ is a hyperelliptic fibration, then the relative canonical map of $f$ is a generically two-to-one map and its proper image is a birationally ruled surface over $C$. Hence, we see that $X$ is birationally equivalent to a double covering of a ruled surface over $C$ (cf. [1, Theorem III 4], [5, Lemma 1.1]).

In order to prove Theorem 0.3 , we find required fibrations whose structures are double coverings of Hirzebruch surfaces. In Section 1, we recall the theory of double coverings of surfaces (cf. [3], 7], [5]). By using this theory, for any positive integers $m$ and a sufficiently large integer $g$, we can prove that there exists no relatively minimal hyperelliptic fibration with $g(f)=g, \chi(f)=g m$ and $\lambda(f)=4$. In consequence of this fact, we see the following: let $z(X)$ be a polynomial in a variable $X$. If there exists a relatively minimal hyperelliptic fibration $f$ with $g(f)=g, \chi(f) \geq z(g)$ and slope four for any positive integer $g$, then the degree of $z(X)$ is greater than one. It follows that Theorem 0.3 is best with respect to the degree of the right side of the inequality.

In Section 2, we give a certain effective divisor which is a branch divisor of a double covering on the $d$-th Hirzebruch surface $\Sigma_{d}$. We call a point $P$ in a divisor $D$ a 2 -fold $j$-ple point if an infinitely near point of $P$ is an ordinary $j$-ple point of the strict transform of $D$ by blowing up at $P$. By applying a method similar to that used in [7. Proposition 3.1] for the purpose of constructing fibrations of genus two, we find sets of suitable effective divisors $B_{k}(k=1,2, \ldots, g+1)$ on $\Sigma_{d}$ such that $B=\sum_{k=1}^{g+1} B_{k}$ has $l_{j} 2$-fold $j$-ple points for $\left(l_{1}, l_{2}, \ldots, l_{g+1}\right) \in \mathbb{Z}_{>0}^{g+1}$ satisfying $\sum_{\alpha=1}^{g+1} j l_{j}=2 d(g+1)$. Considering a double covering branched along $B$, we obtain a hyperelliptic fibration over the projective line. From the theory of a double covering in Section 1 , we give the numerical condition of $\left(l_{1}, l_{2}, \ldots, l_{g+1}\right)$ that the slope of this fibration is equal to four.

In Sections 3, 4 and 5, to conclude the proof Theorem 0.3 we give elements of $\mathbb{Z}_{>0}^{g+1}$ satisfying this numerical condition and compute the Euler-Poincaré characteristic.

\section{Resolution of DOUble COVER AND ITS INVARIANTS}

In this section, we recall the terminology and results on double coverings (cf. 3], [7]). Let $\pi=\pi_{0}: X \longrightarrow Y$ be a double cover between a normal surface $X=X_{0}$ and a smooth algebraic surface $Y=Y_{0}$. Let $B=B_{0}$ be the branch divisor of $\pi$. Denote the rational function field of $X$ by $K(X)$.

Let $\varphi_{0}^{\prime}: Y_{1} \longrightarrow Y_{0}$ be the blowup at a singular point $p_{0}$ of $B$ and $\pi_{1}: X_{1} \longrightarrow Y_{1}$ the $K(X)$-normalization of $Y_{1}$. Then we obtain the natural birational morphism $\varphi_{0}: X_{1} \longrightarrow X_{0}$. Denote the branch divisor of $\pi_{1}$ by $B_{1}$. Continuing this process until the branch divisor $B_{n}$ of $\pi_{n}$ has no singular points, we obtain the sequence of birational morphisms $\varphi_{0}, \varphi_{1}, \ldots, \varphi_{n-1}$ and the following diagram: 


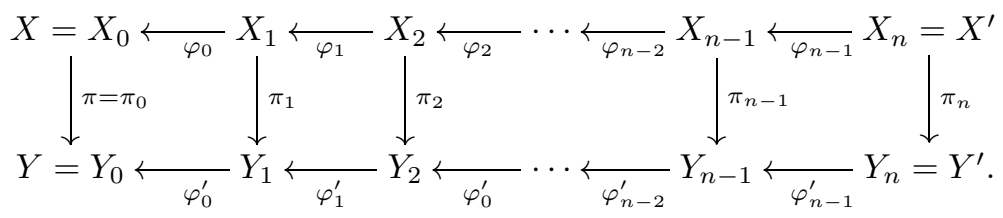

Since $B_{n}$ has no singular points, $X^{\prime}$ is smooth; i.e., the composition $\varphi: X^{\prime} \longrightarrow X$ of morphisms $\varphi_{0}, \varphi_{1}, \ldots, \varphi_{n-1}$ is a resolution of singularities of $X$.

We call such a $\varphi$ the canonical resolution of $\pi$. Denote the multiplicity of $B_{k}$ at $p_{k}$ by $\operatorname{mult}_{p_{k}}\left(B_{k}\right)$ and let $E_{k}$ be the exceptional curve of $\varphi_{k}^{\prime}$. The exceptional curve $E_{k}$ is contained in the branch divisor of $\pi_{k+1}$ if and only if $\operatorname{mult}_{p_{k}}\left(B_{k}\right)$ is even. Hence, $B_{k+1}=\varphi^{\prime *} B_{k}-2\left[\operatorname{mult}_{p_{k}}\left(B_{k}\right) / 2\right] E_{k}$, where $[a]$ is the greatest integer not exceeding a real number $a$. From [3, Lemma 6] and [7, Corollary 2.2], we can compute $\chi\left(\mathcal{O}_{X^{\prime}}\right)$ and $K_{X^{\prime}}^{2}$.

Lemma 1.1 (Horikawa [3, Lemma 6], Persson [7, Corollary 2.2]). Under the same notation as above, denote $\left[\operatorname{mult}_{p_{k}}\left(B_{k}\right) / 2\right]$ by $m_{k}$. Then $X^{\prime}$ has the following numerical properties:

$$
\begin{aligned}
\chi\left(\mathcal{O}_{X^{\prime}}\right) & =2 \chi\left(\mathcal{O}_{Y}\right)+\frac{1}{8} B^{2}+\frac{1}{4} K_{Y} \cdot B-\sum_{k=0}^{n-1} \frac{1}{2} m_{k}\left(m_{k}-1\right), \\
K_{X^{\prime}}^{2} & =2 K_{Y}^{2}+\frac{1}{2} B^{2}+2 K_{Y} \cdot B-\sum_{k=0}^{n-1} 2\left(m_{k}-1\right)^{2} .
\end{aligned}
$$

Proof. See [5, Lemma 1.3, Lemma 1.4].

Note that some $(-1)$-curves may appear on $X^{\prime}$. If these images by $\pi \circ \varphi$ are points of $Y$, then $\varphi$ is not the minimal resolution of $X$. For example, if the branch divisor $B$ has a 2 -fold triple point, then a $(-1)$-curve occurs on $X^{\prime}$.

By using Lemma 1.1, we see the following:

Proposition 1.2. For any positive integer $m$ and a sufficiently large integer $g$, there exists no relatively minimal hyperelliptic fibration $f$ from a surface of general type onto a smooth algebraic curve with $g(f)=g, \chi(f)=g m / 2$ and $\lambda(f)=4$.

Proof. Suppose that there exists a relatively minimal hyperelliptic fibration $f$ from a surface $\widetilde{X}$ of general type onto a smooth algebraic curve $C$ with $g(f)=g, \chi(f)=$ $g m / 2$ and $\lambda(f)=4$ for a sufficiently large integer $g$. Then we may assume that $f$ is the relatively minimal model of the canonical resolution of a double cover $\pi: X \longrightarrow$ $Y$, where $Y$ is a ruled surface over $C$ (cf. [1, Theorem III 4], [5, Lemma 1.1]).

Let $p: Y \longrightarrow C$ be a projection and $\varphi: X^{\prime} \longrightarrow X$ the canonical resolution of $\pi$ described as before. Note that $f: \widetilde{X} \longrightarrow C$ is a relatively minimal model of $p \circ \pi \circ \varphi: X^{\prime} \longrightarrow C$. More precisely, $\widetilde{X}$ coincides with the surface obtained by contacting all (-1)-curves contained in fibers of $p \circ \pi \circ \varphi$. Let $H$ be the tautological divisor of $Y$ and $F$ a certain fiber of $p$. We assume that the branch divisor $B$ of $\pi$ is linearly equivalent to $2(g+1) H+2 n F$. Since we have $\chi(p \circ \pi \circ \varphi)=\chi\left(\mathcal{O}_{X^{\prime}}\right)-\Delta$ 
and $K_{X^{\prime} / C}^{2}=K_{X^{\prime}}^{2}-8 \Delta$, we obtain

$$
\begin{gathered}
\chi(f)=\chi(p \circ \pi \circ \varphi)=\frac{g(g+1)}{2} H^{2}+g n-\sum_{k=0}^{n-1} \frac{1}{2} m_{k}\left(m_{k}-1\right), \\
K_{\widetilde{X} / C}^{2}=K_{X^{\prime} / C}^{2}+\epsilon=2\left(g^{2}-1\right) H^{2}+4(g-1) n-2 \sum_{k=0}^{n-1}\left(m_{k}-1\right)^{2}+\epsilon,
\end{gathered}
$$

where $\epsilon$ is the number of $(-1)$-curves contained in fibers of $p \circ \pi \circ \varphi$.

From 4, V, p. 746], we may assume that $m_{k} \leq[(g+2) / 2] \leq(g+3) / 2$ for all $k$. Denote the integer $(g+1) H^{2}+2 n$ by $\alpha$. From the above equations, we obtain

$$
\begin{aligned}
& \sum_{k=0}^{n-1} m_{k}\left(m_{k}-1\right)=g(\alpha-m), \\
& \sum_{k=0}^{n-1}\left(m_{k}-1\right)^{2} \geq(g-1) \alpha-g m .
\end{aligned}
$$

By the assumption that $m_{k} \leq(g+3) / 2$, we have $\left(m_{k}-1\right) / m_{k} \leq(g+1) /(g+3)$. Hence we obtain

$$
\begin{aligned}
(g-1) \alpha-g m & \leq \sum_{k=0}^{n-1}\left(m_{k}-1\right)^{2} \leq \frac{g+1}{g+3} \sum_{k=0}^{n-1} m_{k}\left(m_{k}-1\right) \\
& =\frac{g(g+1)(\alpha-m)}{(g+3)}
\end{aligned}
$$

i.e., $\alpha \leq 2 g m /(g-3)$. Since $\alpha$ is an integer, this shows that $\alpha \leq 2 m$ for a sufficiently large integer $g$. Moreover, we have

$$
\begin{aligned}
\sum_{k=0}^{n-1}\left(m_{k}-1\right) & =\sum_{k=0}^{n-1}\left\{m_{k}\left(m_{k}-1\right)-\left(m_{k}-1\right)^{2}\right\} \\
& \leq g(\alpha-m)-(g-1) \alpha+g m=\alpha ;
\end{aligned}
$$

i.e., $m_{k} \leq \alpha+1$ for all $k$. By a similar argument as above, we have $g(\alpha-m) \leq$ $\alpha(\alpha+1)$. Hence we obtain $g(\alpha-m) \leq 2 m(2 m+1)$. By our assumption that $g \gg 0$, we have $\alpha=m$ and $m_{k}=1$ for all $k$; i.e., $X$ has at worst rational double points. Then it is easy to see that there exist no $(-1)$-curves contained in a fiber of $p \circ \pi \circ \varphi$. Hence we have $\chi(f)=g m / 2$ and $K_{\widetilde{X} / C}^{2}=2(g-1) m$. This contradicts $\lambda(f)=4$.

Let $z(X)$ be a polynomial in one variable $X$ satisfying the following property:

(*) There exists a relatively minimal hyperelliptic fibration $f$ with $g(f)=$ $g, \chi(f)=z$ and $\lambda(f)=4$ for any positive integers $g$ and $z$ such that $z \geq z(g)$.

If the degree of $z(X)$ is one, then there exists an integer $m$ such that $m g / 2 \geq z(g)$ for a sufficiently large integer $g$. Therefore, from Proposition 1.2, the degree of $z(X)$ satisfying the property $(*)$ is greater than one. On the other hand, from Theorem 0.3 the polynomial $X^{2}+X / 2-1$ satisfies the property $(*)$. Therefore, we see that the lower bound of the degree of the polynomial with the property $(*)$ is two. 


\section{Double Covering of Hirzebruch surface}

In this section, we construct hyperelliptic fibrations which are double coverings of Hirzebruch surfaces for the proof of Theorem 0.3 . Let $p_{d}: \Sigma_{d}=\mathbb{P}_{\mathbb{P}^{1}}\left(\mathcal{O}_{\mathbb{P}^{1}} \oplus\right.$ $\left.\mathcal{O}_{\mathbb{P}^{1}}(d)\right) \longrightarrow \mathbb{P}^{1}$ be the $d$-th Hirzebruch surface with $d \geq 1$. Set $H_{0}^{(d)}=\mathbb{P}_{\mathbb{P}^{1}}\left(\mathcal{O}_{\mathbb{P}^{1}}\right) \subset$ $\Sigma_{d}$ and $H_{\infty}^{(d)}=\mathbb{P}_{\mathbb{P}^{1}}\left(\mathcal{O}_{\mathbb{P}^{1}}(d)\right) \subset \Sigma_{d}$. First, for constructing the branch divisor, we show the existence of the following sets of curves and consider the sum of these curves.

Lemma 2.1. Let $\left(l_{1}, l_{2}, \ldots, l_{g+1}\right)$ be an element of $\mathbb{Z}_{>0}^{g+1}$ with $\sum_{j=1}^{g+1} j l_{j}=2 d(g+1)$. Then there exists a set $\left\{C_{k}\right\}_{k=1}^{g+1}$ of curves on $\Sigma_{2 d}$ satisfying the following conditions:

(i) $C_{k}$ is linearly equivalent to $H_{0}^{(2 d)}$ and is a transversal to $H_{0}^{(2 d)}$.

(ii) The divisor $\sum_{k=1}^{g+1} C_{k}$ has $l_{j}$ ordinary $j$-ple points on $H_{0}^{(2 d)}$; i.e.,

$$
l_{j}=\sharp\left\{P \in H_{0}^{(2 d)} \mid P \text { is an ordinary } j \text {-ple point of } \sum_{k=1}^{g+1} C_{k}\right\} \quad(j=1,2, \ldots, g+1) .
$$

(iii) The divisor $\sum_{k=1}^{g+1} C_{k}$ has at worst double points except for singular points on $H_{0}^{(2 d)}$.

Proof. For integers $i$ and $j$ with $1 \leq i \leq l_{j}$ and $1 \leq j \leq g+1$, we set distinct points $P_{i, j}$ on $H_{0}^{(2 d)}$. In order to obtain the required curves, we give curves $C_{k}$ satisfying the following conditions:

- $C_{k}$ is linearly equivalent to $H_{0}^{(2 d)}$.

- $\left.C_{k}\right|_{H_{0}^{(2 d)}}$ is reduced.

- $j=\sharp\left\{C_{k} \mid C_{k}\right.$ passes through $\left.P_{i, j}\right\}$.

Note that we have $\left.\sum_{k=1}^{g+1} C_{k}\right|_{H_{0}^{(2 d)}}=\sum_{j=1}^{g+1} \sum_{i=1}^{l_{j}} j P_{i, j}$ for these curves.

Since the multiplicities of $\sum_{j=1}^{g+1} \sum_{i=1}^{l_{j}} j P_{i, j}$ at $P_{i, j}$ are less than or equal to $g+1$, we can choose the reduced divisor $D_{k}(k=1,2, \ldots, g+1)$ on $H_{0}^{(2 d)}$ such that $\operatorname{deg} D_{k}=2 d$ and $\sum_{k=1}^{g+1} D_{k}=\sum_{j=1}^{g+1} \sum_{i=1}^{l_{j}} j P_{i, j}$. Let $\mathcal{L}_{k}$ be a sublinear system consisting of divisors $Q \in\left|H_{0}^{(2 d)}\right|$ such that the intersection points of $Q$ and $H_{0}^{(2 d)}$ coincide with the support of $D_{k}$. From the Riemann-Roch Theorem, we have $\operatorname{dim} \mathcal{L}_{k}=1$; i.e., there exist curves $C_{k}$ satisfying $\left.C_{k}\right|_{H_{0}^{(2 d)}}=D_{k}$. From $\sum_{k=1}^{g+1} D_{k}=$ $\sum_{j=1}^{g+1} \sum_{i=1}^{l_{j}} j P_{i, j}$, we obtain

$$
j=\sharp\left\{D_{k} \mid D_{k} \text { contains } P_{i, j}\right\}=\sharp\left\{C_{k} \mid C_{k} \text { passes through } P_{i, j}\right\} .
$$

Hence, it follows that there exist curves $C_{k}$ satisfying the above conditions.

Moreover, by $\left(H_{0}^{(2 d)}\right)^{2}=2 d$, we see that there exists no base point of $\mathcal{L}_{k}$ except for points contained in $D_{k}$. Therefore, we can choose curves $C_{k} \in \mathcal{L}_{k}$ satisfying that $C_{k}$ is a transversal to another $C_{k^{\prime}}$ on $H_{0}^{(2 d)}$ and that $\sum_{k=1}^{g+1} C_{k}$ has at worst double points except for singular points on $H_{0}^{(2 d)}$. Therefore, we obtain the required curves $C_{k}$.

Let $f_{d}: \Sigma_{d} \longrightarrow \Sigma_{2 d}$ be the double cover branched along $H_{0}^{(2 d)}+H_{\infty}^{(2 d)}$. Note that $2 H_{0}^{(d)}=f_{d}^{*} H_{0}^{(2 d)}$. For any element $\left(l_{1}, l_{2}, \ldots, l_{g+1}\right) \in \mathbb{Z}_{>0}^{g+1}$ satisfying the condition $\sum_{\alpha=1}^{g+1} j l_{j}=2 d(g+1)$, we choose a set $\left\{C_{k}\right\}_{k=1}^{g+1}$ of curves as in Lemma 2.1. The effective divisor $B=f_{d}^{*} \sum_{k=1}^{g+1} C_{k}$ is linearly equivalent to $2(g+1) H_{0}^{(d)}$. By the 
conditions (ii) and (iii) in Lemma $2.1, B$ has $l_{j}$ 2-fold $j$-ple points of $B$ on $H_{0}^{(d)}$ and at worst double points except for $H_{0}^{(d)}$. Moreover, analytic branches of singular points of $B$ on $H_{0}^{(d)}$ are tangent to a fiber of $p_{d}$.

Since $B$ is 2-divisible, we can consider the double cover $\pi: X \longrightarrow \Sigma_{d}$ branched along $B$. Let $\varphi: X^{\prime} \longrightarrow X$ be the canonical resolution of $\pi$ given by the following diagram (cf. Section 1):

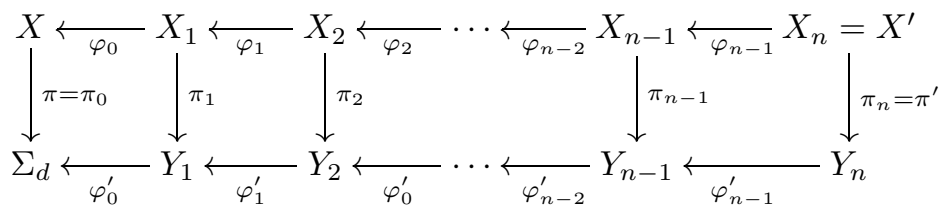

Let $h: \tilde{X} \longrightarrow \mathbb{P}^{1}$ be the relatively minimal model of $p_{d} \circ \pi \circ \varphi$. Then $h$ is a hyperelliptic fibration of genus $g$. In the following, we call this hyperelliptic fibration $h$ a fibration associated to $\left(l_{1}, l_{2}, \ldots, l_{g+1}\right)$. By using Lemma [1.1] we have the following:

Lemma 2.2. Let $\left(l_{1}, l_{2}, \ldots, l_{g+1}\right)$ be an element of $\mathbb{Z}_{>0}^{g+1}$ with $\sum_{j=1}^{g+1} j l_{j}=2 d(g+1)$. Under the same notation as above, the fibration $h: \tilde{X} \longrightarrow \mathbb{P}^{1}$ has the following numerical properties:

$$
\begin{aligned}
\chi(h) & =\frac{d}{2} g(g+1)-\frac{1}{4} \sum_{j=3}^{g+1}\left(j^{2}-2 j\right) l_{j}-\frac{1}{4} \sum_{j \geq 3, j: \text { odd }} l_{j}, \\
K_{\widetilde{X} / \mathbb{P}^{1}}^{2} & =2 d\left(g^{2}-1\right)-\sum_{j=3}^{g+1}(j-2)^{2} l_{j} .
\end{aligned}
$$

Proof. With the notation concerning the canonical resolution of $\pi$ as in Section 1, we first compute $\chi\left(\mathcal{O}_{X^{\prime}}\right)$ and $K_{X^{\prime}}$. Let $P$ be a 2 -fold $j$-ple point of $B$. Then $\left\{\varphi_{i}\right\}_{i=0}^{n-1}$ contains blowups at $P$ and an infinitely near point of $P$. For simplicity, we assume that $\varphi_{0}^{\prime}$ is a blowup at $P$ and that $\varphi_{1}^{\prime}$ is a blowup at an infinitely near point of $P$. Let $E_{0}$ and $E_{1}$ be strict transforms of exceptional curves of $\varphi_{0}^{\prime}$ and $\varphi_{1}^{\prime}$, respectively.

We argue the canonical resolution $\varphi$ of $\pi$ by using Figures 1 and 2 , To illustrate the canonical resolution of $\pi$, thin curves are used to represent components of the branch divisor of $\varphi^{\prime}$ and broken curves are used to represent curves not contained in the branch divisor. Thick curves are used to represent strict transforms of curves in $X^{\prime}$. The self-intersection number is written near the curves.

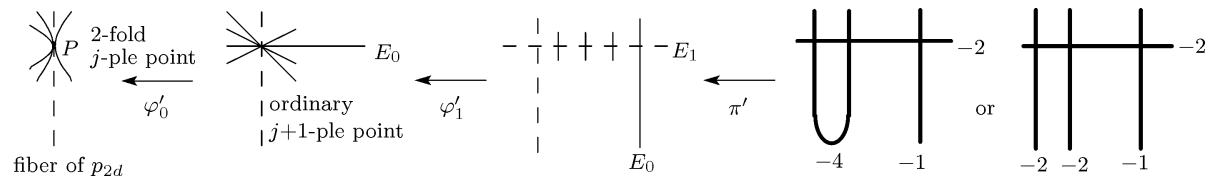

FiguRE 1. In the case that $j$ is odd. 


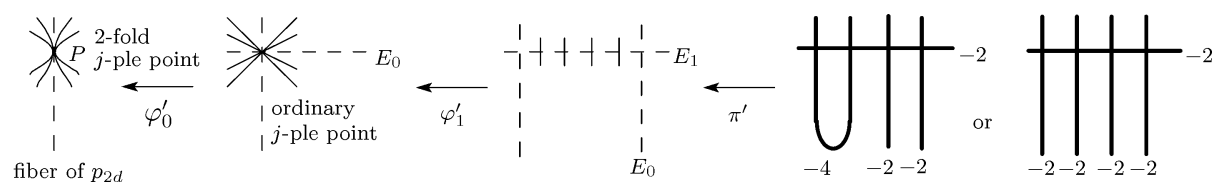

Figure 2. In the case that $j$ is even.

By Figures 1 and 2, we see that

$$
m_{0}=\left\{\begin{array}{ll}
\frac{j-1}{2} & (j: \text { odd }) \\
\frac{j}{2} & (j: \text { even })
\end{array}, \quad m_{1}=\left\{\begin{array}{ll}
\frac{j+1}{2} & (j: \text { odd }) \\
\frac{j}{2} & (j: \text { even })
\end{array} .\right.\right.
$$

The branch divisor $B$ has $l_{j} 2$-fold $j$-ple points on $H_{0}^{(d)}$ and has at worst double points on $\Sigma_{d} \backslash H_{0}^{(d)}$. Hence, by Lemma 1.1, we have

$$
\begin{aligned}
\chi\left(\mathcal{O}_{X^{\prime}}\right) & =2 \chi\left(\mathcal{O}_{\Sigma_{d}}\right)+\frac{B^{2}}{8}+\frac{B \cdot K_{\Sigma_{d}}}{4}-\sum_{j \geq 3, j \text { :even }} \frac{j(j-2)}{4} l_{j}-\sum_{j \geq 3, j \text { :odd }} \frac{(j-1)^{2}}{4}, \\
K_{X^{\prime}}^{2} & =2 K_{\Sigma_{d}}^{2}+\frac{B^{2}}{2}+2 B \cdot K_{\Sigma_{d}}-\sum_{j \geq 3, j \text { :even }}(j-2)^{2} l_{j}-\sum_{j \geq 3, j \text { :odd }}\left\{(j-2)^{2}+1\right\} l_{j} .
\end{aligned}
$$

Since $\chi\left(\mathcal{O}_{\Sigma_{d}}\right)=1, K_{\Sigma_{d}}^{2}=8, B^{2}=4(g+1)^{2} d$ and $B \cdot K_{\Sigma_{d}}=-2(g+1) d-4(g+1)$, we obtain

$$
\begin{aligned}
\chi\left(\mathcal{O}_{X^{\prime}}\right) & =1-g+\frac{(g+1)^{2} d}{2}-\frac{(g+1) d}{2}-\sum_{j=3}^{g+1} \frac{j(j-2)}{4} l_{j}-\sum_{j \geq 3, j \text { :odd }} \frac{l_{j}}{4}, \\
K_{X^{\prime}}^{2} & =8(1-g)+2(g+1)^{2} d-4(g+1) d-\sum_{j=3}^{g+1}(j-2)^{2} l_{j}-\sum_{j \geq 3, j \text { :odd }} l_{j} .
\end{aligned}
$$

Denote the morphism $p_{d} \circ \pi \circ \varphi$ by $h^{\prime}$. Since $h$ is the relatively minimal model of $h^{\prime}$, for computing $\chi(h)$ and $K_{X / \mathbb{P}^{1}}^{2}$, we count the number of $(-1)$-curves in fibers of $h^{\prime}$.

Since $B$ does not contain fibers of $p_{d},(-1)$-curves in fibers of $h^{\prime}$ coincide with certain exceptional curves of $\varphi$. There exists a $(-1)$-curve contained in the inverse image of a 2-fold $j$-ple point by $\pi \circ \varphi$ for an odd integer $j$. (See Figure1) Moreover, we see that there exist no $(-1)$-curves in the exceptional set of $\varphi$ except for these $(-1)$-curves. (See Figure 2.) Hence, the number of $(-1)$-curves in fibers of $h^{\prime}$ is equal to $\sum_{j \geq 3, j \text { :odd }} l_{j}$, i.e., $\chi\left(\mathcal{O}_{X}\right)=\chi\left(\mathcal{O}_{X^{\prime}}\right)$ and $K_{X}^{2}=K_{X^{\prime}}^{2}+\sum_{j \geq 3, j \text { :odd }} l_{j}$. Furthermore, we have $\chi\left(\mathcal{O}_{X}\right)=\chi(h)+1-g$ and $K_{X}^{2}=K_{X / \mathbb{P}^{1}}^{2}+8(1-g)$. We conclude from these equations that

$$
\begin{aligned}
\chi(h) & =\frac{d g(g+1)}{2}-\sum_{j=3}^{g+1} \frac{j(j-2)}{4} l_{j}-\sum_{j \geq 3, j: \text { odd }} l_{j} / 4, \\
K_{X / \mathbb{P}^{1}}^{2} & =2 d\left(g^{2}-1\right)-\sum_{j=3}^{g+1}(j-2)^{2} l_{j} .
\end{aligned}
$$


By Lemma 2.2, the slope of a fibration $h$ associated to $\left(l_{1}, l_{2}, \ldots, l_{g+1}\right)$ is equal to four if and only if the following equation holds:

$$
2 d(g+1)=\sum_{j=3}^{g+1}(2 j-4) l_{j}+\sum_{j \geq 3, j \text { :odd }} l_{j} .
$$

For any $\left(l_{3}, l_{4}, \ldots, l_{g+1}\right) \in \mathbb{Z}_{>0}^{g-1}$ satisfying the condition (2.1), since we have $\sum_{j=3}^{g+1}(2 j-4) l_{j}+\sum_{j \geq 3, j \text { :odd }} l_{j} \geq \sum_{j=3}^{g+1} j l_{j}$, we can choose non-negative integers $l_{1}$ and $l_{2}$ satisfying $\sum_{j=1}^{g+1} j l_{j}=2 d(g+1)$. In order to give a hyperelliptic fibration with slope four, it suffices to give an element $\left(l_{3}, l_{4}, \ldots, l_{g+1}\right) \in \mathbb{Z}_{>0}^{g-1}$ satisfying the condition (2.1). Thus, for simplicity of the notation, we call the above fibration $h$ a fibration associated to $\left(l_{3}, l_{4}, \ldots, l_{g+1}\right)$.

For $\left(l_{3}, l_{4}, \ldots, l_{g+1}\right) \in \mathbb{Z}_{>0}^{g+1}$ satisfying the condition (2.1), a fibration $h$ associated to $\left(l_{3}, l_{4}, \ldots, l_{g+1}\right)$ has the following numerical properties:

$$
\begin{aligned}
& g(h)=g, \quad \lambda(h)=4, \\
& \chi(h)=\frac{d\left(g^{2}-1\right)}{2}-\sum_{j=3}^{g+1} \frac{(j-2)^{2} l_{j}}{4} .
\end{aligned}
$$

\section{In the CASE that the GENUS IS EVEN}

In this section, we prove the existence of fibrations as in Theorem 0.3 (i). Giving an element $\left(l_{3}, l_{4}, \ldots, l_{g+1}\right) \in \mathbb{Z}_{>0}^{g-1}$ satisfying the condition (2.1) , we first construct hyperelliptic fibrations of even genus with slope four.

Proposition 3.1. Let $g$ be an even integer which is greater than four, $N$ a nonnegative integer which is less than or equal to $g / 2-2$ and $d$ a positive integer. For any $\left(k_{0}, \ldots, k_{N+1}\right) \in \mathbb{Z}_{\geq 0}^{N+2}$ satisfying $\sum_{i=0}^{N+1} k_{i}=d$, there exists a relatively minimal hyperelliptic fibration $h$ which has the following invariants:

$$
g(h)=g, \quad \lambda(h)=4, \quad \chi(h)=\frac{d}{2}\left\{g^{2}-g-N(N+1)\right\}-\frac{1}{2} \sum_{i=0}^{N+1} i(i+1) k_{i} .
$$

Proof. We set integers $l_{3}, l_{4}, \ldots, l_{g+1}$ as follows:

$$
\begin{aligned}
& l_{N+3-i}=l_{N+4+i}=k_{i} \quad(N \geq 2, i=0,1,2, \ldots, N-2), \\
& l_{2 N+3}=k_{N-1}, \quad l_{2 N+4}=k_{N} \quad(N \geq 1), \\
& l_{2 N+5}=k_{N+1}, \quad l_{i}=0 \quad(i \geq 2 N+6), \\
& l_{3}=d+k_{N}, \quad l_{4}=d\left(\frac{g}{2}-N-2\right)+k_{N-1},
\end{aligned}
$$

where we set $k_{-1}=k_{0}$. By the assumption that $\sum_{i=0}^{N+1} k_{i}=d$, we have

$$
\begin{aligned}
\sum_{j=3}^{g+1}(2 j-4) l_{j}+\sum_{j \geq 3, j: \text { odd }} l_{j}= & \sum_{i=0}^{N-2}\{2(N+3-i)-4+2(N+4+i)-4+1\} k_{i} \\
& +\{2(2 N+3)-4+1\} k_{N-1}+4\left\{d\left(\frac{g}{2}-N-2\right)+k_{N-1}\right\} \\
& +\{2(2 N+4)-4\} k_{N}+3\left(d+k_{N}\right)+\{2(2 N+5)-4+1\} k_{N+1} \\
= & \sum_{i=0}^{N+1}(4 N+7) k_{i}+2 d(g-2 N-4)+3 d=2 d(g+1) .
\end{aligned}
$$


Hence, $\left(l_{3}, l_{4}, \ldots, l_{g+1}\right)$ satisfies the condition (2.1).

Let $h$ be a fibration associated to $\left(l_{3}, l_{4}, \ldots, l_{g+1}\right)$. Since it is clear that $g(h)=g$ and $\lambda(h)=4$, it is enough to compute the relative Euler-Poincaré characteristic of $h$. From the equation (2.2) and the assumption that $\sum_{i=0}^{N+1} k_{i}=d$, we have

$$
\begin{aligned}
\chi(h)= & \frac{d\left(g^{2}-1\right)}{2}-\sum_{i=0}^{N-2}\left\{\frac{(N+1-i)^{2}+(N+2+i)^{2}}{4}\right\} k_{i}-\frac{(2 N+1)^{2}}{4} k_{N-1} \\
& -\frac{(2 N+2)^{2}}{4} k_{N}-\frac{(2 N+3)^{2}}{4} k_{N+1}-d\left(\frac{g}{2}-N-2\right)-k_{N-1}-\frac{d+k_{N}}{4} \\
= & \frac{d\left(2 g^{2}-2 g+4 N+5\right)}{4}-\sum_{i=0}^{N+1}\left\{\frac{2 N^{2}+6 N+5+2 i(i+1)}{4}\right\} k_{i} . \\
= & \frac{d\left(g^{2}-g-N^{2}-N\right)}{2}-\frac{1}{2} \sum_{i=0}^{N+1} i(i+1) k_{i} .
\end{aligned}
$$

For integers $a$ and $b$, we denote the intersection of the interval $[a, b]($ resp. $[a, \infty))$ and $\mathbb{Z}$ by $[a, b]_{\mathbb{Z}}\left(\right.$ resp. $\left.[a, \infty)_{\mathbb{Z}}\right)$. The positive integer denoted by $i(i+1) / 2(i \in$ $\left.\mathbb{Z}_{>0}\right)$ is called a triangular number. Before we prove Theorem 0.3 (i), we show the following lemma from the well-known fact on the representation of a positive integer by a sum of three triangular numbers.

Lemma 3.2. For an integer $d$ which is greater than or equal to three and a positive integer $N$, set

$$
\Delta_{N, d}=\left\{\sum_{i=0}^{N} \frac{1}{2} i(i+1) k_{i} \mid k_{i} \in \mathbb{Z}_{\geq 0}, \sum_{i=0}^{N} k_{i}=d\right\} .
$$

Then $\Delta_{N, d}$ contains $\left[0, d N(N+1) / 2-N^{2}\right]_{\mathbb{Z}}$.

Proof. Let $z$ be an integer which is less than $(N+1)(N+2) / 2$. It is well-known that any positive integer is represented by a sum of at most three triangular numbers. Therefore, $z$ is represented by a sum of at most three triangular numbers which are less than $(N+1)(N+2) / 2$, i.e., $z \in \Delta_{N, 3}$.

Let $m$ and $z^{\prime}$ be integers such that $3 \leq m \leq d$ and $(m-1) N(N+1) / 2-N^{2} \leq$ $z^{\prime}<m N(N+1) / 2-N^{2}+1$. The integer $z^{\prime}-(m-3) N(N+1) / 2$ is positive and less than $(N+1)(N+2) / 2$. Hence, we have $z^{\prime}-(m-3) N(N+1) / 2 \in \Delta_{N, 3}$, i.e., $z^{\prime} \in \Delta_{N, m} \subset \Delta_{N, d}$. Thus, we obtain $\left[0, d N(N+1) / 2-N^{2}\right]_{\mathbb{Z}} \subset \Delta_{N, d}$.

Next we show that the fibration required in Theorem 0.3 (i) coincides with one of the fibrations constructed in Proposition 3.1 by using the previous lemma. For a set $I \subset \mathbb{Z}$ and $x \in \mathbb{Z}$, we denote the set $\{x-z \in \mathbb{Z} \mid z \in I\}$ as $x-I$.

Proposition 3.3. For an even integer $g \geq 6$ and an integer $z \geq g^{2}+g / 2-2$, there exists a relatively minimal hyperelliptic fibration $h$ with $\lambda(h)=4, g(h)=g$ and $\chi(h)=z$.

Proof. By Proposition 3.1, it suffices to prove that an integer $z \geq g^{2}+g / 2-2$ is represented by the form

$$
\frac{1}{2} \sum_{i=0}^{N+1} k_{i}\left(g^{2}-g-N(N+1)-i(i+1)\right),
$$


where the $k_{i}$ 's are non-negative integers and $N$ is an integer such that $0 \leq N \leq$ $g / 2-2$. Denote $\sum_{i=0}^{N+1} k_{i}$ by $d$ and set

$$
R_{g, d, N}=\frac{d}{2}\left(g^{2}-g-N(N+1)\right)-\Delta_{N+1, d} .
$$

Hence it is enough to show that $\bigcup_{d \geq 1} \bigcup_{N=0}^{\frac{g}{2}-2} R_{g, d, N} \supset\left[g^{2}+g / 2-2, \infty\right)_{\mathbb{Z}}$.

By Lemma 3.2, if $d$ is greater than two, then $\Delta_{N+1, d}$ contains $[0, d(N+1)(N+$ $\left.2) / 2-(N+1)^{2}\right]_{\mathbb{Z}}$. Moreover, it is easy to see that $\Delta_{1, d} \supset[0,3 d-2]_{\mathbb{Z}}$. Hence, we have

$$
\begin{aligned}
R_{g, d, N} & \supset\left[d\left(g^{2}-g-2(N+1)^{2}\right) / 2+(N+1)^{2}, d\left(g^{2}-g-N(N+1)\right) / 2\right]_{\mathbb{Z}}, \\
R_{6, d, 1} & \supset[11 d+2,14 d]_{\mathbb{Z}} .
\end{aligned}
$$

Denote $d\left(g^{2}-g-2(N+1)^{2}\right) / 2+(N+1)^{2}$ by $\alpha_{g, d, N}, d\left(g^{2}-g-N(N+1)\right) / 2$ by $\beta_{g, d, N}$. Now we consider the set $\bigcup_{N=0}^{\frac{g}{2}-2} R_{g, d, N}$. In the case that $d \geq 3$, since we have $N \geq 0$, we have

$$
\beta_{g, d, N+1}-\alpha_{g, d, N}+1=\left(\frac{d}{2}-1\right) N^{2}+\left(\frac{d}{2}-2\right) N \geq 0
$$

i.e., we obtain $\alpha_{g, d, N}-1 \in\left[\alpha_{g, d, N+1}, \beta_{g, d, N+1}\right]_{\mathbb{Z}}$ for $d \geq 3$. Hence, it follows that $\bigcup_{N=0}^{\frac{g}{2}-2} R_{g, d, N} \supset\left[\alpha_{g, d, \frac{g}{2}-2}, \beta_{g, d, 0}\right]_{\mathbb{Z}}$ and $\bigcup_{N=0}^{1} R_{6, d, N} \supset\left[11 d+2, \beta_{6, d, 0}\right]_{\mathbb{Z}}$.

If we assume that $d \geq 4$ and $g \geq 8$, then we have

$$
\beta_{g, d-1,0}-\alpha_{g, d, \frac{g}{2}-2}+1=\frac{(g-2)^{2}}{4}\left(d-\frac{3 g}{g-2}\right) \geq \frac{(g-2)(g-8)}{4} \geq 0 .
$$

If we assume that $d \geq 4$ and $g=6$, then we have

$$
\beta_{6, d-1,0}-(11 d+2)+1=4 d-16 \geq 0 .
$$

Therefore, we have $\bigcup_{d \geq 1} \bigcup_{N=0}^{\frac{g}{2}-2} R_{g, d, N} \supset\left[\alpha_{g, 3, \frac{g}{2}-2}, \infty\right)_{\mathbb{Z}}$. Since we have $\alpha_{g, 3, \frac{g}{2}-2}$ $=g^{2}+g / 2-2$, the assertion follows from it.

\section{IN THE CASE THAT THE GENUS IS ODD AND GREATER THAN FIVE}

We need several propositions to prove the existence of fibrations as in Theorem 0.3 (ii) in the case that the genus is odd and greater than five. These propositions are proved by the same calculations as in Proposition 3.1. In the proof of these, we give only integers $l_{3}, l_{4}, \ldots, l_{g+1}$ satisfying the condition (2.1) and do not repeat the same argument concerning invariants of the fibration associated to $\left(l_{3}, l_{4}, \ldots, l_{g+1}\right)$.

Proposition 4.1. Let $g$ be an odd integer which is greater than three, $N$ a nonnegative integer which is less than or equal to $(g-5) / 2$ and $d$ an integer which is greater than two. For any $\left(k_{0}, \ldots, k_{N+1}\right) \in \mathbb{Z}_{\geq 0}^{N+2}$ satisfying $d=\sum_{i=0}^{N+1} k_{i}$, there exists a relatively minimal hyperelliptic fibration $h$ which has the following numerical properties:

$$
g(h)=g, \quad \lambda(h)=4, \quad \chi(h)=\frac{d}{2}\left\{g^{2}-g-N(N+1)\right\}-\frac{1-(-1)^{d}}{2}-\frac{1}{2} \sum_{i=0}^{N+1} i(i+1) k_{i} .
$$


Proof. The following integers $l_{3}, l_{4}, \ldots, l_{g+1}$ satisfy the condition (2.1):

$$
\begin{aligned}
& l_{N+3-i}=l_{N+4+i}=k_{i} \quad(N \geq 2, i=0,1,2, \ldots, N-2), \\
& l_{2 N+3}=k_{N-1}, \quad l_{2 N+4}=k_{N} \quad(N \geq 1), \\
& l_{2 N+5}=k_{N+1}, \quad l_{i}=0 \quad(i \geq 2 N+6), \\
& l_{3}=d-\left\{1-(-1)^{d}\right\}+k_{N}, \quad l_{4}=d\left(\frac{g-4}{2}-N\right)+k_{N-1}+\frac{3}{4}\left(1-(-1)^{d}\right),
\end{aligned}
$$

where we set $k_{-1}=k_{0}$.

Proposition 4.2. Let $g$ be an odd integer which is greater than five, $N$ a nonnegative integer which is less than or equal to $(g-7) / 2$ and $d$ an integer greater than three. For any $\left(k_{0}, \ldots, k_{N+1}\right) \in \mathbb{Z}_{\geq 0}^{N+2}$ satisfying $d=\sum_{i=0}^{N+1} k_{i}$, there exists a relatively minimal hyperelliptic fibration $h$ which has the following numerical properties:

$$
g(h)=g, \quad \lambda(h)=4, \quad \chi(h)=\frac{d}{2}\left\{g^{2}-g+2-N(N+1)\right\}-\frac{1}{2} \sum_{i=0}^{N+1} i(i+1) k_{i} .
$$

Proof. The following integers $l_{3}, l_{4}, \ldots, l_{g+1}$ satisfy the condition (2.1):

$$
\begin{aligned}
& l_{N+3-i}=l_{N+4+i}=k_{i} \quad(N \geq 2, i=0,1,2, \ldots, N-2), \\
& l_{2 N+3}=k_{N-1}, \quad l_{2 N+4}=k_{N} \quad(N \geq 1), \\
& l_{2 N+5}=k_{N+1}, \quad l_{i}=0 \quad(i \geq 2 N+6), \\
& l_{3}=3 d+k_{N}, \quad l_{4}=d\left(\frac{g-7}{2}-N\right)+k_{N-1},
\end{aligned}
$$

where we set $k_{-1}=k_{0}$.

Proposition 4.3. Let $g$ be an odd integer which is greater than five. For any element $\left(k_{0}, \ldots, k_{\frac{g-3}{2}}\right)$ in $\mathbb{Z}_{\geq 0}^{\frac{g-1}{2}}$ satisfying $\sum_{i=0}^{\frac{g-3}{2}} k_{i}=4$, there exists a relatively minimal hyperelliptic fibration $h$ which has the following numerical properties:

$$
g(h)=g, \quad \lambda(h)=4, \quad \chi(h)=\frac{3}{2} g^{2}-\frac{7}{2}-\frac{1}{2} \sum_{i=0}^{\frac{g-3}{2}} i(i+1) k_{i} .
$$

Proof. The following integers $l_{3}, l_{4}, \ldots, l_{g+1}$ satisfy the condition (2.1):

$$
\begin{aligned}
& l_{\frac{g+3}{2}-i}=l_{\frac{g+5}{2}+i}=k_{i} \quad\left(i=0,1,2, \ldots, \frac{g-7}{2}\right), \\
& l_{g}=k_{\frac{g-5}{2}}, \quad l_{g+1}=k_{\frac{g-3}{2}}, \quad l_{3}=k_{\frac{g-3}{2}}, \quad l_{4}=k_{\frac{g-5}{2}}+1 .
\end{aligned}
$$

Proposition 4.4. Let $g$ be an odd integer which is greater than five. For any element $\left(k_{0}, \ldots, k_{\frac{g-3}{2}}\right)$ in $\mathbb{Z}_{\geq 0}^{\frac{g-1}{2}}$ satisfying $\sum_{i=0}^{\frac{g-3}{2}} k_{i}=3$, there exists a relatively minimal hyperelliptic fibration $h$ which has the following numerical properties:

$$
g(h)=g, \quad \lambda(h)=4, \quad \chi(h)=\frac{9}{8} g^{2}-\frac{17}{8}-\frac{1}{2} \sum_{i=0}^{\frac{g-3}{2}} i(i+1) k_{i} .
$$


Proof. The following integers $l_{3}, l_{4}, \ldots, l_{g+1}$ satisfy the condition (2.1):

$$
\begin{aligned}
& l_{\frac{g+3}{2}-i}=l_{\frac{g+5}{2}+i}=k_{i} \quad\left(i=0,1,2, \ldots, \frac{g-7}{2}\right), \\
& l_{g}=k_{\frac{g-5}{2}}, \quad l_{g+1}=k_{\frac{g-3}{2}}, \quad l_{3}=k_{\frac{g-3}{2}}+1, \quad l_{4}=k_{\frac{g-5}{2}} .
\end{aligned}
$$

The fibration required in Theorem 0.3 (ii) coincides with one of the fibrations constructed in Propositions 4.144.4. We prove the following proposition by an argument similar to the proof of Proposition 3.3

Proposition 4.5. For an odd integer $g \geq 7$ and an integer $z \geq g^{2}-1$, there exists a relatively minimal hyperelliptic fibration $h$ with $\lambda(h)=4, g(h)=g$ and $\chi(h)=z$.

Proof. We set

$$
\begin{aligned}
S_{g, d, N} & =\frac{d}{2}\left\{g^{2}-g-N(N+1)\right\}-\frac{1-(-1)^{d}}{2}-\Delta_{N+1, d} \quad\left(0 \leq N \leq \frac{g-5}{2}\right), \\
T_{g, d, N} & =\frac{d}{2}\left\{g^{2}-g+2-N(N+1)\right\}-\Delta_{N+1, d} \quad\left(0 \leq N \leq \frac{g-7}{2}\right), \\
U_{g} & =\frac{3}{2} g^{2}-\frac{7}{2}-\Delta_{\frac{g-3}{2}, 4}, \\
V_{g} & =\frac{9}{8} g^{2}-\frac{17}{8}-1-\Delta_{\frac{g-3}{2}, 3} .
\end{aligned}
$$

From Propositions 4.1,4.4, it suffices to show that a positive integer $z$ satisfying $z \geq g^{2}-1$ is contained in one of the sets $S_{g, d, N}, T_{g, d, N}, U_{g}$ and $V_{g}$. In other words, we show that $\bigcup_{d \geq 1}\left(\bigcup_{N=1}^{(g-5) / 2} S_{g, d, N} \cup \bigcup_{N=1}^{(g-7) / 2} T_{g, d, N}\right) \cup U_{g} \cup V_{g} \supset\left[g^{2}-1, \infty\right)_{\mathbb{Z}}$.

Denote $d\left(g^{2}-g\right) / 2-(d-1)(N+1)^{2}$ and $d\left(g^{2}-g-N(N+1)\right) / 2$ by $\alpha_{g, d, N}$ and $\beta_{g, d, N}$, respectively. From Lemma 3.2. if $d$ is greater than two, then we have

$$
\begin{aligned}
S_{g, d, N} \supset\left[\alpha_{g, d, N}-\frac{1-(-1)^{d}}{2}, \beta_{g, d, N}-\frac{1-(-1)^{d}}{2}\right]_{\mathbb{Z}}, \\
T_{g, d, N} \supset\left[\alpha_{g, d, N}+d, \beta_{g, d, N}+d\right]_{\mathbb{Z}}, \\
U_{g} \supset\left[\frac{5 g^{2}+2 g-11}{4}, \frac{3 g^{2}-7}{2}\right]_{\mathbb{Z}}, \\
V_{g} \supset\left[g^{2}-1, \frac{9 g^{2}-17}{9}\right]_{\mathbb{Z}} .
\end{aligned}
$$

If we assume that $d \geq 3$, then

$$
\beta_{g, d, N+1}-\alpha_{g, d, N}+1=\left(\frac{d}{2}-1\right) N^{2}+\left(\frac{d}{2}-2\right) N \geq \frac{N(N-1)}{2} .
$$

Hence, we obtain

$$
\bigcup_{N=0}^{\frac{g-5}{2}} S_{g, d, N} \cup \bigcup_{N=0}^{\frac{g-7}{2}} T_{g, d, N} \supset\left[\alpha_{g, d, \frac{g-5}{2}}-\frac{1-(-1)^{d}}{2}, \beta_{g, d, 0}+d\right]_{\mathbb{Z}} \quad(d \geq 3) .
$$

Moreover, since we have $\Delta_{1, d} \supset[0,3 d-2]_{\mathbb{Z}}$ for $d \geq 3$, we obtain $\left(\bigcup_{N=0}^{1} S_{7, d, N}\right) \cup$ $T_{7, d, 1} \supset[17 d+2,22 d]_{\mathbb{Z}}$.

If we assume that $d \geq 5$, then we have

$$
\left(\beta_{g, d-1,0}+d-1\right)-\alpha_{g, d, \frac{g-5}{2}}+1 \geq \frac{(g-4)(g-7)}{2} \geq 0 .
$$


Therefore, we obtain

$$
\bigcup_{d \geq 4}\left(\bigcup_{N=0}^{\frac{g-5}{2}} S_{g, d, N} \cup \bigcup_{N=0}^{\frac{g-7}{2}} T_{g, d, N}\right) \supset\left[\gamma_{g}, \infty\right)_{\mathbb{Z}},
$$

where $\gamma_{g}$ is equal to $\alpha_{g, 4, \frac{g-5}{2}}$ if $g \geq 9$ and is equal to 70 if $g=7$.

Since we have

$$
\begin{aligned}
\frac{3 g^{2}-7}{2}-\gamma_{g}+1 & =\left\{\begin{array}{cl}
\frac{g^{2}-10 g+17}{4} \geq 0 & \text { if } g \geq 9, \\
1 & \text { if } g=7,
\end{array}\right. \\
\left(\beta_{g, 3,0}+3\right)-\frac{5 g^{2}+2 g-11}{4}+1 & =\frac{g^{2}-8 g+27}{4} \geq 0, \\
\frac{9 g^{2}-17}{8}-\left(\alpha_{g, 3, \frac{g-5}{2}}-1\right)+1 & =\frac{g^{2}-12 g+35}{8} \geq 0,
\end{aligned}
$$

it follows that

$$
\bigcup_{d \geq 3}\left(\bigcup_{N=0}^{\frac{g-5}{2}} S_{g, d, N} \cup \bigcup_{N=0}^{\frac{g-7}{2}} T_{g, d, N}\right) \cup U_{g} \cup V_{g} \supset\left[g^{2}-1, \infty\right)_{\mathbb{Z}} .
$$

\section{In the CASE that the Genus IS FIVE}

In this section, we prove the existence of fibrations as in Theorem 0.3 (ii) in the case that the genus is five by an argument similar to Proposition 4.5. We prepare the following:

Proposition 5.1. Let $d$ be a positive integer. For any $\left(k_{0}, k_{1}, k_{2}\right) \in \mathbb{Z}_{\geq 0}^{2}$ satisfying $\sum_{i=0}^{2} k_{i}=d$, there exists a relatively minimal hyperelliptic fibration $h$ which has the following numerical properties:

$$
g(h)=5, \lambda(h)=4, \chi(h)=11 d-2 \sum_{i=0}^{2} i k_{i} .
$$

Proof. The following integers $l_{3}, l_{4}, \ldots, l_{g+1}$ satisfy the condition (2.1):

$$
l_{3}=4 k_{0}, \quad l_{4}=3 k_{1}+k_{2}, \quad l_{5}=0, \quad l_{6}=k_{2} .
$$

Then the fibrations associated to $\left(l_{3}, l_{4}, \ldots, l_{g+1}\right)$ satisfy the requirements.

Proposition 5.2. For an integer $z \geq 20$, there exists a relatively minimal hyperelliptic fibration $h$ with $\lambda(h)=4, g(h)=5$ and $\chi(h)=z$.

Proof. We use the same notation as in the proof of Proposition 4.5 and set

$$
W_{d}=\left\{z \in[7 d, 11 d]_{\mathbb{Z}} \mid z \equiv d \bmod 2\right\} .
$$

From Propositions 4.4 and 5.1, for any positive integer $z \in\left(\bigcup_{d \geq 1} W_{d}\right) \cup V_{5}$, there exists a relatively minimal hyperelliptic fibration $h$ with $g(h)=5, \lambda(h)=4$ and $\chi(h)=z$. Hence, we show that $\left(\bigcup_{d \geq 1} W_{d}\right) \cup V_{5} \supset[20, \infty)_{\mathbb{Z}}$. 
By an easy calculation, we have

$$
\begin{gathered}
\bigcup_{d \geq 3 \text { :odd }} W_{d}=\left\{z \in[21, \infty)_{\mathbb{Z}} \mid z \text { is odd }\right\}, \\
\bigcup_{d \geq 3 \text { :even }} W_{d}=\left\{z \in[28, \infty)_{\mathbb{Z}} \mid z \text { is even }\right\} .
\end{gathered}
$$

Now we have $\bigcup_{d \geq 3} W_{d}=[27, \infty)_{\mathbb{Z}}$. We obtained $V_{5} \supset[24,26]_{\mathbb{Z}}$ in the proof of Proposition 4.5. Moreover, we have 20, $22 \in W_{2}$ and $21,23 \in W_{3}$, and we see that $\left(\bigcup_{d \geq 1} W_{d}\right) \cup V_{5} \supset[20, \infty)_{\mathbb{Z}}$. This completes the proof of the proposition.

From Propositions 4.5 and 5.2 , we have Theorem 0.3 (ii).

\section{ACKNOWLEDGMENTS}

The author expresses his thanks to Professor Tadashi Ashikaga for valuable discussions and advice. Thanks are also due to Professors Nobuo Hara and Mizuho Ishizaka for encouragement and advice.

\section{REFERENCES}

1. A. Beauville, Surfaces algébriques complexes, Astérisque 54 (1978). Soc. Math. France. MR0485887 (58:5686)

2. W. Barth, K. Hulek, C. Peters, A. Van de Ven, Compact complex surfaces, 2nd Edition, Springer-Verlag, Berlin, 2004. MR2030225 (2004m:14070)

3. E. Horikawa, On deformations of quintic surfaces, Invent Math. 31 (1975), 43-85.

4. E. Horikawa, Algebraic surfaces of general type with small $c_{1}{ }^{2}$, I, II, III, IV, V, Ann. of Math. (2) 104 (1976), 358-387; Invent. Math. 37 (1976), 121-155; ibid. 47 (1978), 209-248; ibid 50 (1978/79), 103-128; J. Fac. Sci. Univ. Tokyo 28 (1981), 745-755. MR0424831 (54:12789) MR0460340 (57:334) MR0501370 (80h:14012a) MR0517773 (80h:14012b) MR0656051 (84d:14019)

5. H. Ishida, Bound of the relative Euler-Poincaré characteristic for certain hyperelliptic fibrations, Manuscr. Math. 118 (2005), 467-483. MR2190108 (2006j:14012)

6. K. Konno, Clifford index and the slope of fibered surfaces, J. Algebraic Geom. 8 (1999), no. 2, 207-220. MR 1675150(2000e:14060)

7. U. Persson, Double coverings and surfaces of general type, Lect. Notes in Math., 687, Springer, 1978, 168-195. MR527234(80h:14017)

8. U. Persson, Chern invariants of surfaces of general type, Comp. Math. 43 (1981), 3-58. MR631426 (83b:14012)

9. G. Xiao, Fibered algebraic surfaces with low slope, Math. Ann. 276 (1987), 449-466. MR875340 (88a:14046)

Ube National College of Technology, 2-14-1 Tokiwadai, Ube 755-8555, Yamaguchi, JAPAN

E-mail address: ishida@ube-k.ac.jp 